\title{
智能起重机的关键特征和控制系统总体架构
}

\author{
李国杰 \\ (苏州大学应用技术学院 苏州 215325)
}

\begin{abstract}
摘要: 起重机正在朝着智能化方向发展, 起重机的智能化特征和控制系统架构决定了智能起重机的总体性能和技术方向, 是 起重机智能化研究的核心。简要回顾了我国起重机产品从传统起重机到智能起重机的发展历程, 比较了传统起重机、自动化 起重机、工业机器人和智能起重机的主要性能特征和关联性, 分析了我国起重机智能化研究应重点关注的行业和起重机产品, 对智能起重机的通用性和个性化关键特征进行了分析和总结。提出了智能起重机控制系统的总体架构, 对系统架构中各关键 功能模块和各子系统进行了详细分析阐述, 讨论了各模块及子系统之间的关联性、主要数据流向及起重机智能化实现方法, 强调了构建强大的内、外部信息互联、顺畅的数据交换和共享机制对智能起重机的重要性, 定义起重机广域智能系统, 包括 智能起重机在内的广域智能系统的群体智能化是实现智能起重机系统智能化作业的关键, 而且广域智能系统信息共享和数据 互联互通的深度和广度决定了系统智能化的水平。
\end{abstract}

关键词：智能起重机；关键特征；控制系统；总体架构；数据互联

中图分类号: $\mathrm{TH} 21$

\section{Key Characteristics and General Framework of Control System for Intelligent Crane}

\author{
LI Guojie
}

(Applied Technology College, Soochow University, Suzhou 215325)

\begin{abstract}
Crane is developing towards the intelligent direction. The intelligent characteristics and the control system framework determine the overall performance and technical direction of intelligent crane, which is the core of crane intellectualization research. The development process of China's crane products from traditional crane to intelligent crane is briefly reviewed, and the main performance characteristics and relevance of traditional crane, automatic crane, industrial robot and intelligent crane are compared. The related industries and crane products that should be focused on in the intelligent crane research is proposed. The universality and personalization features of intelligent crane are analyzed and summarized. Moreover the general framework of intelligent crane control system is put forward, and the key functional modules and subsystems is analyzed in detail. The relevance between the modules and subsystems, the main data flow direction and the realization method of intelligent crane are discussed. The importance of the construction of a powerful internal and external information interconnection, smooth data exchange and sharing mechanism of intelligent crane system are emphasized. The wide-area intelligent system of crane is defined for the first time. And the group intelligence of wide-area intelligent system including the intelligent crane is the key to realize intelligent operation of intelligent crane system, and the depth and breadth of information sharing and data interconnection of the wide-area intelligent system determine the level of intelligent system.
\end{abstract}

Key words: intelligent crane; key characteristics; control system; general framework; data interconnectionk

0 前言

新一代信息技术的快速发展正在深刻影响并改

20200320 收到初稿, 20200715 收到修改稿
变着我国传统制造业，2015 年《中国制造 2025》正 式发布 ${ }^{[1]}$, 在智能制造已成为国家战略的大背景下, 智能工厂和智慧港口等智能化升级工程得到快速推 进, 我国智能装备产业将面临难得的发展机遇。2019 年 11 月我国交通运输部发布的《关于建设世界一流 港口的指导意见》明确要求, 到 2025 年我国部分沿 
海集装箱枢纽港初步形成全面感知、泛在互联、港 车协同的智能化系统, 2035 年集装箱枢纽港基本建 成智能化系统。岸边集装箱起重机和场地集装箱起 重机等产品是集装箱枢纽港关键核心起重设备, 这 些起重设备的智能化技术水平将直接影响我国智慧 港口等重大建设项目的顺利实施。因此, 包括智能 化港口起重机在内的智能起重机的开发及其关键技 术研究将成为行业近年重点研究和攻关方向。

目前我国在智能起重机领域的研究处于起步阶 段, 由于起重机行业产品利润率普遍不高, 并且多 为单件小批量定制生产模式, 多数企业为了首先解 决企业生存问题, 不得不将主要研发力量投入到非 标合同产品开发设计工作中, 起重机共性基础研究、 关键核心技术及高端产品开发总体投入不足, 导致 国内起重机行业前沿性关键技术积累不多, 包括智 能起重机在内的创新研究能力受到一定限制。

此外, 我国目前尚无可以规范并引领智能起重 机研究和产品开发、设计及制造的通用性标准, 部 分行业企业对智能起重机关键特征、起重机智能化 实现方法、智能控制系统的组成和关键功能模块等 方面的认识尚存在一定差异, 无法形成关键核心技 术的研究合力, 一定程度影响了我国智能起重机产 品的技术进步和稳健发展。

我国在起重机自动化控制方面已经取得了一定 的研究和应用成果。宓为建等 ${ }^{[2]}$ 应用机器视觉等方 法解决了集装箱锁孔识别和自动定位问题; 杨宇华 等 ${ }^{[3-7]}$ 围绕集装箱港口及港口起重机产品的自动化 开展了系列研究; 李国杰 ${ }^{[8-9]}$ 以实现起重机搬运作业 自动化为目标, 构建了搬运物有初始摆动和无初始 摆动两种作业状态下的摆动控制系统; 李永等 ${ }^{[10]} 介$ 绍了一种核电厂电机组装用全自动起重机, 该全自 动起重机已经在核电厂投入使用。除了港口和核电 外, 一些具有自动化作业功能的起重机产品已经在 仓储、钢铁、能源等行业得到应用。智能起重机是 建立在起重机自动化控制技术基础上, 应用新一代 信息通信技术对起重机现有技术进行系统升级, 使 起重机具有智能感知、数据分析、优化决策、自适 应控制、实时监控、自评估自诊断、信息共享和通 用网络互联等能力的高性能起重机械, 因此, 起重 机自动化控制技术作为起重机智能化研究的基础, 将会在智能起重机的研究中发挥有效作用。

日本是较早开展起重机自动化控制研究和应用 的国家之一, 北森 ${ }^{[11]}$ 在 1964 年首次以实现起重机作 业自动化为目标开展了起重机搬运物摆动控制的研 究, 此后起重机搬运物摆动控制研究成为起重机自
动化控制重要的研究方向并得到全球起重机研究者 的重点关注。森下嚴 ${ }^{[12]}$ 提出一种时间轴逆转反馈控 制方法, 通过模型试验确认该摆动控制方法的有效 性; 近藤良等 ${ }^{[13-14]}$ 分别基于摆动周期和采用遗传算 法开展搬运物摆动控制研究; 此外, SMOCZEK 等 ${ }^{[15]}$ 提出一种基于模糊逻辑和极点配置法的摆动 控制技术和基于立体视觉系统的起重机工作空间识 别方法; ROGER 等 ${ }^{[16]}$ 介绍了一种考虑钢丝绳长度 变化因素的控制器设计方法; MAGHSOUDI 等 ${ }^{[17]}$ 提出一种改进的单自由度摆动控制器, 可以有效适 应钢丝绳长度的动态变化; ANDRES 等 ${ }^{[18]}$ 提出一种 防止搬运物摆动并可以快速定位搬运物的控制技 术; RAMLI 等 ${ }^{[19]}$ 全面回顾并总结 2000-2016 年 16 年间全球起重机系统控制的研究成果和控制策略, 发现有大量起重机搬运物摆动控制方面的研究文 献, 而且, 这些研究的目标正在从自动化转向智能 化, 并没有发现对起重机系统的控制策略和架构进 行全面综述的文章。

除了起重机搬运物摆动控制研究外, KANESGIGE 等 ${ }^{[20]}$ 提出一种构建起重机自主作业系 统的方法, 该方法包含在线障碍物识别、路径规划 和搬运物摆动控制技术; RINCON 等 ${ }^{[21}$ 提出一种基 于机器学习和人工神经网络的起重机逆动态控制仿 真方法, 仿真试验结果显示具有良好控制性能; DUONG 等 ${ }^{[2]}$ 采用神经网络技术对塔式起重机控制 系统开展研究; SZPYTKO 等 ${ }^{[23]}$ 开展了基于数字孪 生技术的港口集装箱起重机综合维修决策研究, 并 通过实例验证该研究的有效性。对起重机自动化、 智能化研究现状分析后认为, 全球起重机主流研究 方向正在逐步从自动化向智能化方向转变, 起重机 智能化研究已经得到行业研究者普遍关注并正在成 为起重机产品重要的研究领域。

工业机器人、汽车和机床等行业在智能化研究 领域起步较早。汪首坤等 ${ }^{[24]}$ 提出了一种基于混合群 智能优化的机器人立体视觉三步标定方法, 满足机 器人精密视觉操作的需求; 季旭全等 ${ }^{[25]}$ 以实现装配 过程的智能化与精准化为目标, 提出一种基于机器 人与视觉引导的星载设备智能装配方法, 实现 $0.3 \mathrm{~mm}$ 的装配精度; 刘久富等 ${ }^{[26]}$ 针对码垛机器人作 业环境状况复杂、不确定条件多的问题, 采用多智 能体 $\mathrm{Q}$ 值算法对多关节码垛机器人进行了路径规划 研究, 有效提升作业精度; 史也等 ${ }^{[27}$ 提出一种可使 基座姿态和机械臂末端位姿同时到达期望状态的路 径规划方法, 通过仿真试验对该规划方法进行了验 证; LEGASHEV 等 ${ }^{[28]}$ 提出一种基于云的自主机器 
人智能系统监测体系结构; 贾庆轩等 ${ }^{[29]}$ 利用 $\mathrm{A} *$ 算法 在空间机械臂的自由工作空间进行无碰撞路径搜 索, 实现空间机械臂的避障路径规划。李运华等 ${ }^{[30]}$ 就多轴驱动车辆的路径跟踪控制开展了研究; 张利 鹏等 ${ }^{[31]}$ 提出并验证了一种结合主动转向与差动转 向的分布式驱动智能汽车双重转向控制方法, 全面 改善了车辆的通过性和方向稳定性; 江浩斌等 ${ }^{[32]}$ 提 出一种基于距离和视觉信息融合的停车位智能识别 方法, 提升了车位智能识别准确率; 章仁鲛等 ${ }^{[33}$ 通 过对智能汽车转向系统进行主动控制, 获得了准确 的智能汽车转向轮转角; 王跃飞等 ${ }^{[34]}$ 开展了智能汽 车 Ethernet AVB 网络设计与优化研究, 很好地满足 了智能汽车网络高速实时的通信要求; 牛礼民等 [35-36]分别对动力汽车动力总成多智能体集成控制策 略和智能网联汽车路径跟踪控制器进行了研究与设 计; BAHNES 等 ${ }^{[37]}$ 开发一种自主作业车辆之间的通 信系统, 提升港口智能车辆自主作业效率和精度。 熊振华等 ${ }^{[38]}$ 提出了一种智能车床颤振的实时辨识 与在线抑制系统, 实现了车床的智能化颤振抑制; 黄莹等 ${ }^{[39-42]}$ 分别就云计算在智能机床中的应用、切 削过程中的智能技术、智能机床的状态监测诊断以 及智能机床高端切削刀具等开展研究。姜洪开等 ${ }^{[43]}$ 提出了一种基于深度学习的飞行器智能故障诊断方 法, 有效建立监测数据与关键机械部件健康状态间 的精确映射联系, 实现准确的故障诊断和预测。康 荣杰等 ${ }^{[44]}$ 引出了机械智能的概念, 总结了传统机 构、柔性机构及机器人中所展现的机械智能, 并认 为机械智能以其系统可靠性、快速响应性和人机交 互安全性等优点必将成为今后智能机器或机器人发 展的重要方向之一。这些智能化领域的研究成果值 得起重机智能化研究借鉴。

本文简要回顾我国起重机产品从传统起重机到 智能起重机的发展历程, 对传统起重机、自动化起 重机、工业机器人和智能起重机的主要性能特征进 行了对比, 简要分析 4 类产品的关联和区别。分析 了智能起重机潜在的应用领域和重点应关注的产品 类别, 总结了智能起重机的关键特征, 提出了智能 起重机控制系统总体架构, 并对主要功能模块和子 系统进行详细阐述。

\section{1 从传统起重机到智能起重机的发展}

我国制造业经历了传统制造、先进制造和建立 在信息化基础上的高端制造的不同发展阶段, 并正 在快速向智能制造方向发展。起重机作为制造业重
要的物流装备, 同样经历了从传统起重机、半自动 起重机到自动化起重机的发展过程。伴随着制造业 智能化的步伐, 智能起重机将会成为制造业进入智 能制造大时代后急需发展并需重点突破的关键智能 装备之一, 起重机行业正在逐步迈向智能起重机的 新时代。

为了对智能起重机产品的关键特征有比较清晰 的认识, 先将起重机在不同发展阶段的产品性能特 征进行分析对比。

\section{1 从传统起重机到自动化起重机的发展过程}

起重机是传统制造业特别是机械装备制造业不 可或缺的关键物流设备。传统起重机的显著特征之 一是只有由司机操控才能完成正常的搬运作业, 司 机操控起重机的方式包括在设置于起重机上的司机 室内和在地面通过线控或遥控等方式进行。由于传 统起重机进行搬运作业时, 一般需要地面工作人员 配合进行搬运物装载挂钩和卸载脱钩等协助作业, 起重机司机的操作指令也需要通过与地面工作人员 的互动获取, 因此, 传统起重机存在作业效率低、 作业成本高、安全性差等不足。

20 世纪 80 年代起, 为了降低生产成本和提高 生产效率，我国传统制造业加快了自动化升级改造 步伐, 对工厂关键配套设备提出越来越高的自动化 作业要求, 实现起重机搬运作业的半自动化或自动 化成为满足制造业升级的关键能力。

自动化立体仓库用巷道堆垛起重机等产品是较 早实现作业自动化的起重机产品, 由于该类起重机 产品的作业装置与起重机主体用刚性方式连接, 可 以实现较高的搬运物作业定位精度, 因此在制造业 自动化升级中得到广泛应用。包括工厂通用桥、门 式起重机和港口集装箱起重机等在内的多数起重机 产品, 由于其作业装置与起重机主体采用钢丝绳柔 性连接, 起重机作业过程中搬运物存在不同程度的 垂直摆动和水平回转运动, 这种搬运物的不确定性 运动状态影响并干扰了起重机的正常搬运和快速定 点卸载等作业; 同时, 由于该类起重机的作业装置 在无外界干预情况下处于柔性状态, 难以满足起重 机对搬运物的自动装卸作业和三维定位精度等要 求, 因此, 这类起重机没有在传统制造业自动化升 级改造初期发挥出显著的自动化作业能力并得到普 遍应用。

在汽车及其零部件、制药、日用家电等行业的 工厂自动化升级改造中, 大量采用工业机器人取代 起重机的部分功能, 进行物料的装卸和位置转移作 业, 满足了物料搬运作业自动化要求, 实现了工厂 
自动化生产链的完整和稳定运行。

工业机器人作为重要的自动化物流设备在工厂 自动化改造升级中得到普遍应用, 是因为工业机器 人可以自动完成搬运物的装载和卸载, 快速完成搬 运物任意角度倾斜和上下翻转, 并可以与相邻机器 人自动完成搬运物交接, 具有敏捷的移动速度和对 搬运物精准的定位等能力。此外, 通过调整程序指 令, 工业机器人可以完成不同工位、不同工艺要求 的作业任务, 实现一机多用。

工业机器人所拥有的这些性能特征和作业精准 程度, 通用起重机产品尚无法等同实现。因此, 在 搬运物重量和搬运作业范围较小的工厂自动化改造 中, 工业机器人成为物料搬运作业的主力设备并得 到广泛应用, 工业机器人在推进工厂自动化建设中 发挥了积极作用。

工业机器人与起重机相比, 主要不足是承载能 力弱、作业范围小, 不适合在大起重量、高起升高 度和长水平搬运距离的搬运作业场合作业, 因此无 论制造业处于哪个发展阶段, 工业机器人均无法代 替起重机。

\section{2 自动化起重机现状及与智能起重机的主要区别}

经过科研攻关和技术积累, 我国在起重机半自 动化和自动化控制方面取得了阶段性研究成果, 并 在机械装备制造、物料仓储以及集装箱港口等行业 中得到推广应用, 实现了起重机自动化或半自动化 连续搬运作业, 取得了良好的应用效果。

自动化起重机是在传统起重机基础上进行自动 化技术升级的起重机产品, 其目的是实现起重机搬 运作业的无人化, 通常应用于流程性很强的单一重 复性工作的作业场所。自动化起重机可以根据管理 人员输入的作业指令, 通过自动检测、信息采集、 信息处理和分析判断, 自动完成预期的物料搬运作 业任务。

我国制造业已从传统制造迈向了智能制造时 代, 但并不意味着我国制造业已经走完了智能制造 前面的全部发展历程。事实上, 由于我国工业基础 比较弱, 电气化和信息化发展阶段的基础尚有待进 一步夯实, 在关键、核心零部件开发和基础技术研 究等方面仍然有许多关键技术需要持续开展科技攻 关并予以解决。同样地, 虽然我国现有的自动化作 业起重机在处理和控制起重机确定性的工况、流程 和作业要求等方面已积累了一定经验, 但距离客户 期待的自动化作业精度、作业安全性、装卸效率和 作业成本等性能指标的要求还有一定差距。特别是 起重机的作业装置与起重机主体采用钢丝绳柔性连
接的这类起重机，虽然在搬运物摆动控制等技术上 取得一定的研究和应用性成果, 但由于该类起重机 的主体与作业装置处于柔性关联状态, 包括搬运物 自动装卸、搬运物位置状态调整和精准定位、搬运 物与工厂内其他设备以及在制品等的防碰撞安全控 制以及搬运路径规划等在内的关键技术和科研成果 尚不能完全满足自动化流水线对起重机作业精度和 性能的要求。

智能起重机是在自动化起重机基础上进行智能 化升级的高性能起重机, 与自动化作业起重机比较, 最大区别在于自动化起重机必须接受管理者输入的 作业指令才能进行作业, 也就是说, 自动化起重机 虽然无须人直接操作就可以自动作业, 但归根到底 是通过人来控制的。而智能起重机具有显著的工业 4.0 技术特征, 不仅可以满足作业安全性、作业生产 率、作业质量、可重复性和成本控制等起重机搬运 作业性能指标要求, 而且具有从失败或人为干预中 学习和复原的能力 ${ }^{[45-47]}$, 可以像人一样理性行动和 自主作业。

各阶段起重机产品以及工业机器人关键性能和 特征对比分析如表 1 所示。

表 1 各阶段起重机产品及工业机器人性能特征对比

\begin{tabular}{ccccc}
\hline 产品 & $\begin{array}{c}\text { 传统 } \\
\text { 起重机 }\end{array}$ & $\begin{array}{c}\text { 自动化 } \\
\text { 起重机 }\end{array}$ & $\begin{array}{c}\text { 工业 } \\
\text { 机器人 }\end{array}$ & $\begin{array}{c}\text { 智能 } \\
\text { 起重机 }\end{array}$ \\
\hline 作业特征 & 司机 & 指令 & 指令 & 自主 \\
起重能力 & 大 & 大 & 小 & 大 \\
作业范围 & 大 & 大 & 小 & 大 \\
产品成本 & 低 & 中 & 中偏高 & 高 \\
作业成本 & 高 & 中 & 中偏低 & 低 \\
作业效率 & 低 & 中 & 中偏高 & 高 \\
作业精度 & 低 & 中 & 高 & 中偏高 \\
\hline
\end{tabular}

起重机智能化控制技术建立在物联网、人工智 能和大数据等新一代信息技术和起重机自动化控制 技术研究成果的基础上，具有十分明显的跨学科结 构特征。面对起重机自动化技术基础尚未完全夯实 的客观现实, 加之起重机作业环境非模式、不确定 性因素的影响, 迎接起重机行业全面智能化时代的 到来还需要全行业科技工作者继续开展科研攻关并 付出艰苦努力。

\section{3 起重机智能化研究应重点关注的行业和产品}

起重机根据其服务的行业和具体作业用途的不 同有多种类型，不同类型起重机产品的结构形式、 构造特点、作业方式和作业工艺等均存在一定差异。 受起重机服务行业的市场规模大小、发展水平以及 对起重机自动化、智能化水平要求的高低等因素的 
影响, 不同类型的起重机产品的技术水平差别较大, 产品的个性化特征也比较明显。

起重机智能化研究的目标并不是要实现所有类 型起重机的智能化, 而应重点关注对起重机智能化 有迫切需求的行业, 以及这些行业重点关注的起重 机产品类型。以市场需求为导向, 最大可能满足市 场需求是智能起重机关键技术研究的目标和方向。

一般来讲, 自动化升级起步早、发展快、自动化技 术和经验积累较多的行业, 其智能化发展的迫切感 也比较强烈。因此, 建议首先聚焦自动化发展水平 比较高, 智能化发展潜力比较大的行业, 在此基础 上关注这些行业智能化建设对起重机产品类别和智 能化水平的具体需求, 有了行业和市场清晰的产品 智能化要求, 起重机智能化研究的针对性会更强,

目的性也会更明确。

进入 21 世纪后, 诞生于汽车制造业的自动化流 水线生产模式正在被越来越多的制造企业采纳和运 用, 伴随着产品及零部件的数字化、标准化、模块 化和生产工艺的不断成熟, 制造业自动化、智能化 迎来前所未有的发展机会。汽车制造业也由于其拥 有比较成熟的自动化体系, 将最有可能成为我国智 能制造战略实施的第一块试验田。

目前汽车制造业自动化流水线普遍采用工业机 器人进行工厂物料搬运和转移作业, 随着汽车制造 业先进的生产模式向其他制造业转移和智能工厂建 设的深入推进, 可以取代工业机器人, 具有大起重 能力、高定位精度和广作业范围的工厂智能化起重 机将成为包括汽车业在内的机械制造业普遍关注的 关键智能装备, 也是未来需要重点开展智能化技术 攻关的关键起重机产品之一。

自动化立体仓库是自动化水平较高的仓储设 备, 也是汽车自动化流水线关键自动化设备, 已经 在包括汽车工业在内的机械制造业、通用基础零部 件、医药、电子和航空航天等行业得到广泛应用。 巷道堆垛起重机作为自动化立体仓库的关键自动化 设备, 是在起重机行业比较早期开展自动化技术研 究与应用的产品, 该类产品具有良好的自动化应用 和智能化升级的基础, 值得重点关注。

随着上海洋山港四期自动化码头 2017 年 10 月 正式开港运行, 我国在全球港口自动化领域的研究 开发与应用取得里程碑式成果, 为 2035 年实现我国 集装箱枢纽港智能化系统建设目标打下了良好的基 础。岸边集装箱起重机和场地集装箱起重机等集装 箱枢纽港关键起重设备的智能化发展将面临难得的 发展机遇。
此外, 还应该重点关注能源、钢铁、物流仓储 以及航空航天等行业现有自动化设备的智能化升级 需求, 以及需要在高温、高污染等特殊环境条件下 作业的起重机的自动化、智能化需求, 包括垃圾搬 运起重机、钢厂钢卷和板坏搬运起重机、铸造起重 机、自动搬运小车等在内的自动化起重机产品已经 得到很好应用, 这些产品也是近年应重点关注并投 入力量进行智能化升级研究的起重机产品。

智能起重机与其服务的行业以及具体的智能化 系统之间具有密切的关联性, 本文将包括智能起重 机在内的智能工厂或智慧港口等智能化系统定义为 广域智能系统。

\section{2 智能起重机的关键特征}

智能起重机的特征可以分为通用性特征和个性 化特征两类, 通用性特征是所有智能起重机均拥有 的产品特征, 而个性化特征取决于广域智能系统对 特定智能起重机产品的个性化作业需求, 不具有普 遍性。智能起重机所拥有的个性化特征和能力往往 决定了智能化产品的竞争力和创新力, 值得研究者 关注。

智能起重机是在传统起重机和自动化起重机基 础上发展起来的高性能起重机, 因此, 传统起重机 和自动化起重机所拥有的基本性能特征同样适用于 智能起重机，后续将不再赘述。在传统起重机向自 动化起重机发展过程中, 由关键、核心技术支撑的 关键性能特征, 将在起重机智能化发展过程中融入 新的技术和研究成果, 并得到进一步提升和强化。

\section{1 智能起重机的通用性关键特征}

智能起重机具有自我监测和感知能力, 能够感 知并自动获取起重机自身的作业能力、作业环境条 件、作业场地设备和物料、起重机装卸作业过程和 被搬运货物的运动状态等支撑智能起重机正常作业 所需的所有数据和信息; 具有符合标准通信接口的 信息共享机制和顺畅的数据互联互通、实时快速交 换处理能力; 具有学习和解决新问题的能力, 通过 变换处理、建模分析、大数据挖掘处理和行动决策, 自动生成起重机智能化作业的工作指令, 使起重机 能像人一样理性、自主作业。

监测感知、数据互联、学习分析、优化决策、 实时反馈、自适应、理性行动和像人一样自主作业 等构成了智能起重机比较典型的通用性关键特征。

\subsection{1 智能起重机的主要数据及其特征}

多数起重机的起升机构通常采用柔性钢丝绳连 
接取物装置进行货物的搬运作业, 起重机装卸作业 现场环境状态复杂多变, 起重机与相邻起重机、起 重机与广域智能系统中静止和移动的设备和物料之 间, 均存在诸多相互关联又相互影响的因素。起重 机在比较复杂的环境条件下实现柔性作业系统中搬 运物的三维位置精准定位等作业控制属于非线性、 非模式的控制问题, 不仅无法用数学模型描述, 而 且没有一个固定的模式。实现起重机作业的智能化, 非模式、不确定性问题是最大的困扰, 解决这些不 确定问题成为保障可预期装卸作业性能结果的 关键。

数据是认识并最终解决起重机智能化控制中非 模式、不确定性问题的基础。感知、采集并分析起 重机作业全过程中的相关数据, 可以发现凭经验无 法发现或意识不到的影响起重机装卸作业的因素和 各影响因素之间存在的相互关联, 可以降低认知的 不确定性。因此, 数据的有效利用和数据的广域互 联成为实现起重机智能化的关键。

智能起重机正常作业需要大量内置数据和动态 数据支持, 除了在开发、制造过程中保存在系统内 部和出厂时植入控制系统的数据库和知识库数据 外, 智能起重机的主要动态数据包括感知、分析、 决策、执行和自适应等五类数据。

智能起重机的感知数据包括内感知数据和外感 知数据, 内感知数据从智能起重机内部获取, 包括 起重机在作业全过程中的位移、速度、加速度等运 动状态数据、起重机的起重量等作业载荷感知数据、 起重机结构疲劳损坏程度、各机构传动和制动系统 磨损状态等安全性的自诊断感知数据以及起重机有 效作业能力和有效作业范围等自身能力的自感知数 据等; 外感知数据从智能起重机外部获取, 包括起 重机作业现场设备与目标搬运物形状特征和位置状 态、目标搬运物移动和动态变化趋势、作业现场温 度、湿度、海拔高度等地理气象等的感知数据。

智能起重机的分析、决策、执行和自适应数据 具有很强的交互性和关联性, 上一阶段输出的数据 将成为下一阶段数据的入口, 数据流始终双向或多 向贯穿于智能起重机分析、决策、执行及自适应作 业全的过程。通过包括广域智能系统数据在内的广 域数据信息挖掘、提炼以及基于数据的计算分析和 推理预测, 为智能化起重机提供作业决策。

\subsection{2 智能起重机搬运物的数字信息特征}

使起重机广域智能系统内的搬运物具有可识别 的信息, 并在此基础上建立搬运物与智能起重机之 间顺畅的数据交互机制, 将有利于智能起重机对搬
运物的快速搜寻和对搬运物位置的准确定位, 有效 提升起重机搬运作业效率，降低作业成本。

在广域智能系统内的每件搬运物均具有唯一的 物料码和与物料码互联的数据信息情况下, 广域智 能系统给智能起重机的作业指令中可以通过物料码 信息指定搬运物, 智能起重机通过读取物料码自动 获取货物的重量、外形尺寸等基本信息，通过搜寻 与物料码绑定的搬运物获取搬运物的空间位置、放 置状态以及目标搬运物与周边其它货物的相对位置 关系等信息。这些信息与环境识别系统信息结合构 成智能起重机作业不可或缺的关键信息。

在广域智能系统, 搬运物的物料码是该物料全 生命周期的固定标识, 将伴随该物料从原材料到所 属产品全生命周期结束回收利用的全过程。目前广 域智能系统的搬运物通过标识和编码普遍具备了被 感知的基础和能力, 但搬运物主动感知能力尚未普 遍推行, 使搬运物不仅具有被感知能力而且具有自 感知能力是广域智能系统未来的重要发展方向。万 物世界, 有了数据互联和状态互动, 就留下了无限 的想象空间和所有遐想实现的可能。

\subsection{3 智能起重机具有的人工智能特征}

进入 21 世纪后, 人工智能技术随着经验学习、 深度学习和大数据云计算等技术的应用得到快速发 展, 智能感知、视觉识别、智能算法等人工智能技 术彻底改变了传统装备制造业, 推动了装备智能化 和精细化的发展。

人工智能技术的应用可以使智能起重机具有大 数据分析处理、智能判断和理性行动等智能化特征, 使智能起重机具有自学习、自决策和自适应能力, 实现起重机与广域智能系统之间的实时交互, 保证 了起重机与起重机之间以及起重机与广域智能系统 内所有设备与系统之间的协同和精确配合作业。

人工智能技术还可以通过实时分析包括起重机 结构疲劳损坏程度、实际作业能力、各机构传动和 制动状态等感知数据, 准确预判智能起重机剩余寿 命和整机及零部件工作状况, 实现起重机的预测性 维护和保养, 最大可能发挥起重机作业能力, 提升 作业生产效率。

人工智能等技术为智能起重机进行数据处理分 析以及利用数据解决非模式、不确定性问题提供了 有效方法。起重机智能化研究的本质和主要任务, 就是有效利用人工智能和大数据等现代信息技术, 对现有传统起重机及自动化起重机技术进行智能化 升级研究, 有效解决起重机智能化作业过程中的不 确定性问题, 实现真正意义上的智能化装卸作业。 


\section{2 智能起重机的个性化关键特征}

不同的广域智能系统通常会对智能起重机产品 的构造特点、关键技术参数和作业性能指标提出个 性化要求, 因此, 智能起重机最重要的个性化特征 就是使起重机满足并实现广域智能系统对起重机提 出的个性化作业和智能化要求。此外, 由于智能起 重机具有广域数字互联的特征, 对于与广域智能系 统的数据交互具有较强的依赖性, 因此智能起重机 个性化将是未来主流的发展方向。

起重机根据其取物装置与起重机主体连接方式 的不同可以简单分为两大类: 一类产品的取物装置 与起重机主体通过刚性连接件连接, 简称刚性连接 起重机, 用于工厂自动化立体仓库的巷道堆垛起重 机和用于集装箱港口的集装箱正面吊运起重机等均 属于这类起重机; 另一类产品的取物装置与起重机 主体采用钢丝绳柔性连接, 简称柔性连接起重机, 集装箱港口的岸边集装箱起重机、场地集装箱起重 机和工厂通用桥、门式起重机等均属于柔性连接类 起重机。虽然不同的广域智能系统对智能起重机的 要求会有所区别, 但由于这两类起重机结构和作业 特点的区别, 分别具有各自相对一致的个性化性能 特征。

\subsection{1 刚性连接起重机个性化特征}

与柔性连接起重机相比, 刚性连接起重机在搬 运物三维运动状态控制和货物三维位置准确定位等 方面具有显著的优势。例如, 巷道堆垛起重机作业 装置的定位精度可以达到毫米级水平, 这个定位精 度虽然低于工业机器人微米级的定位精度水平, 但 该类起重机产品不需要采取特别的控制方法就可以 满足定位精确度要求不高的作业要求, 这也是巷道 堆垛起重机在所有起重机产品中较早实现作业自动 化的部分原因。

刚性连接起重机与工业机器人构造特点比较接 近, 具有一定的可比性。除了搬运物定位精度外, 刚性连接起重机在搬运物自动装卸、搬运物任意角 度倾斜或翻转以及快速敏捷的作业能力等方面与工 业机器人比较仍有不小差距, 因此, 刚性连接起重 机应该在发挥自身大起重量、广作业范围突出优势 的同时, 强化对工业机器人产品关键技术的研究和 引用, 通过取长补短, 提升该类产品的综合作业能 力和作业精度水平, 使刚性连接类起重机在智能化 升级中得到更加广泛的应用。

\subsection{2 柔性连接起重机个性化特征}

与刚性连接起重机不同, 搬运物在起重机搬运 作业过程中表现出的柔性不确定运动状态是柔性连
接起重机显著的个性化特征, 由于该类起重机具有 特殊的柔性构造, 与刚性连接起重机相比, 在搬运 物自动抓取、位置状态调整、定点卸载以及安全和 精准作业控制等方面均存在一定差距。

《港口起重机设计规范》对岸边集装箱起重机等 柔性连接港口起重机摆动控制精度有明确要求, 要 求规定, 岸边集装箱起重机进行集装箱吊装作业过 程中，在被吊装的集装箱离开大车轨道面的垂直高 度为 $10 \mathrm{~m}$, 起重机小车以额定速度运行, 制动停车 后 $10 \mathrm{~min}$ 内, 被吊装的集装箱的水平摆动幅度应该 控制在 $\pm 100 \mathrm{~mm}$ 内 $^{[48]}$, 这一作业精度也是岸边集装 箱起重机进行集装箱摆动控制后满足集装箱对箱作 业的基本精度要求。

岸边集装箱起重机的集装箱吊具为 4 点起吊, 与工厂通用桥、门式起重机常规的同轴多滑轮多倍 率单吊点起吊方式相比, 前者搬运物定位性能要优 于后者。因此, 工厂通用桥、门式起重机要实现刚 性连接类起重机毫米级定位精度要求, 有比较大的 难度。柔性连接起重机要满足毫米级作业精度要求 的广域智能系统, 需要在机械结构和控制水平优化 等方面进一步开展研究和攻关。

这里需要说明的是, 起重机智能化研究的主要 目标之一是提高产品搬运作业效率, 因此在满足广 域智能系统对起重机搬运物摆动幅度控制精度等要 求的基础上，如何以最快的速度将搬运物的摆动幅 度控制在目标精度范围内是起重机摆动控制研究的 关键。只要给起重机足够的等待时间, 即使不采取 任何控制措施，搬运物的摆动幅度也会逐步衰减下 来，因此不考虑摆动控制时间的摆动控制精度是没 有实际意义的, 也就是说, 没有效率支撑的高精度 会失去其应有的价值。

\section{3 智能起重机控制系统总体架构}

决定起重机智能化水平的关键是智能起重机的 控制系统，建立在新一代信息技术和人工智能基础 上的智能控制系统创新是未来智能起重机重点研究 方向。

智能起重机控制系统总体架构如图 1 所示, 智 能起重机控制系统由中央控制中心和感知层、数据 交互层、人机交互管理层及广域互联层组成。图 1 中广域智能系统总控中心是包括智能起重机在内的 广域智能系统的控制和管理中心，是智能起重机及 智能起重机控制系统的上一级管控系统, 负责向智 能起重机发送作业指令, 并通过人机交互管理层对 
智能起重机的作业进行管理。

强大的数字互联能力是支撑并实现起重机智 能化的基础。智能起重机对内必须建立各层级功 能模块和所有子系统之间顺畅的数据交换和共享 机制, 对外需要与广域智能系统及广域智能系统
总控中心保持实时信息共享和数据互联, 确保智 能起重机所有的信息均可以被汇总分析、所有的 数据均可以互连互通、起重机所有作业均可协同 互动、起重机各执行机构和所有子系统均可精准 控制。

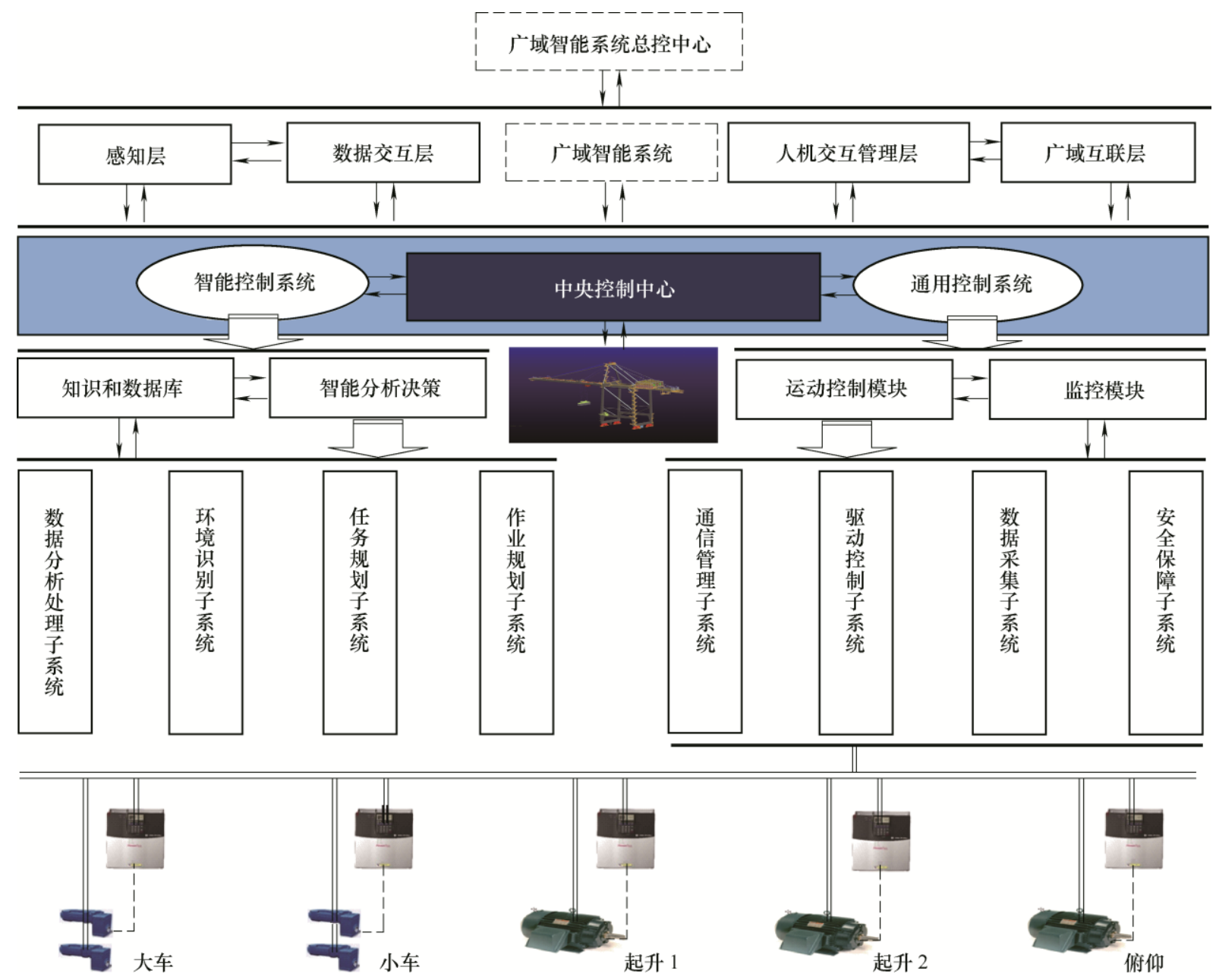

图 1 智能起重机控制系统总体架构

\section{1 中央控制中心}

中央控制中心是智能起重机控制系统的中枢, 是使起重机拥有智能的关键核心系统, 是智能起重 机搬运作业的规划者和控制方案的最终决策者、发 布者和执行者。智能起重机中央控制中心由通用控 制系统和智能控制系统两大系统组成。

\subsection{1 通用控制系统}

通用控制系统接受来自智能控制系统发布的作 业指令, 负责智能起重机作业指令的执行和作业过 程的监控。中央控制中心的通用控制系统保留了传 统起重机的控制系统功能, 在对传统起重机控制系 统进行必要升级并增设起重机智能控制系统和相关 功能模块后, 可以实现传统起重机的智能化升级改 造, 因此通用控制系统是构成智能起重机中央控制 中心的基本控制系统。中央控制中心通用控制系统
包含起重机运动控制和监控两大模块。通用控制系 统总体框架和主要数据流如图 2 所示。

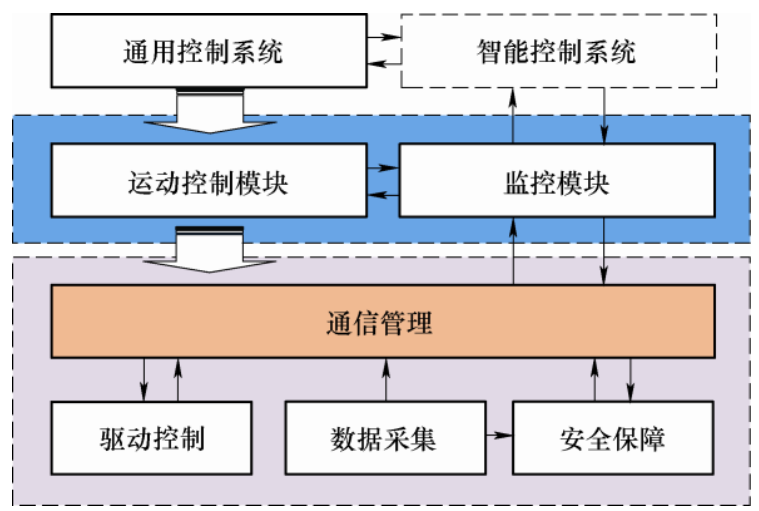

图 2 通用控制系统总体框架和主要数据流

\subsubsection{1 运动控制模块}

运动控制模块由通信管理、驱动控制、数据采 
集、安全保障等子系统组成。

通信管理子系统负责监控模块和运动控制模块 以及运动控制模块所属各子系统之间的数据连接和 通信管理, 通信管理子系统也通过监控模块实现通 用控制系统与智能控制系统之间的数据互联。

接受监控模块的指令, 并将获取的指令实时下 传到驱动控制子系统, 通过驱动系统执行指令任务; 同时, 通信管理子系统还将数据采集子系统采集到 的数据信息实时上传到监控模块。

驱动控制子系统通过通信管理子系统接受来自 监控模块的指令, 根据接收到的工作指令要求驱动 智能起重机大小车运行机构、起升机构、变幅机构 等起重机主要执行机构, 最终通过对智能起重机搬 运作业全过程的有效控制, 实现智能起重机柔性高 效作业。驱动控制子系统也接受并执行监控系统根 据数据采集子系统上传的数据进行分析并下传的局 部路径修正、障碍物紧急回避以及出于安全考虑必 须采取应急响应的特殊指令。

数据采集子系统是构成智能起重机感知系统的 重要组成部分, 主要负责起重机作业状态数据和与 作业安全相关的环境状态数据的采集, 该子系统将 采集到的信息和数据实时上传到监控模块，同时将 与安全关联的信息与数据实时传送给安全保障子 系统。

安全保障子系统接受从数据采集子系统和感知 层获取的作业环境状态、起重机作业状态、搬运物 运动状态以及作业现场障碍物位置和形状等数据, 对起重机与相邻作业设备之间, 以及起重机搬运物 与广域智能系统内其他设备或障碍物之间是否存在 干涉碰撞等问题进行作业安全评估, 并将安全评估 结果通过通信管理子系统上传到监控模块, 监控模 块进行综合分析和判断后通过通信管理子系统向驱 动控制子系统发送局部作业调整指令, 并监督指令 的执行。

\subsubsection{2 监控模块}

监控模块接受和传输智能控制系统发出的控制 指令, 协调智能起重机各执行机构和系统之间的关 系，实现通用控制系统与智能控制系统之间的实时 数据交互, 对智能起重机的作业进行诊断和管理。

监控模块具有数据挖掘和作业误差分析功能, 通过分析和挖掘数据采集子系统和感知层上传的数 据, 结合安全保障子系统上传的安全评估信息, 可 以进行智能起重机局部作业误差修正和避障作业 导航。

监控模块可以通过人机交互管理层实现系统参
数设定、状态变换和数据检测反馈, 能动态监测起 重机的作业状态并实时进行故障诊断和系统报警。

\subsection{2 智能控制系统}

智能控制系统是中央控制中心的核心，是智能 起重机的智慧大脑和控制系统的总控平台, 该系统 由知识库、数据库和智能分析决策模块组成。智能 控制系统总体框架和主要数据流如图 3 所示。

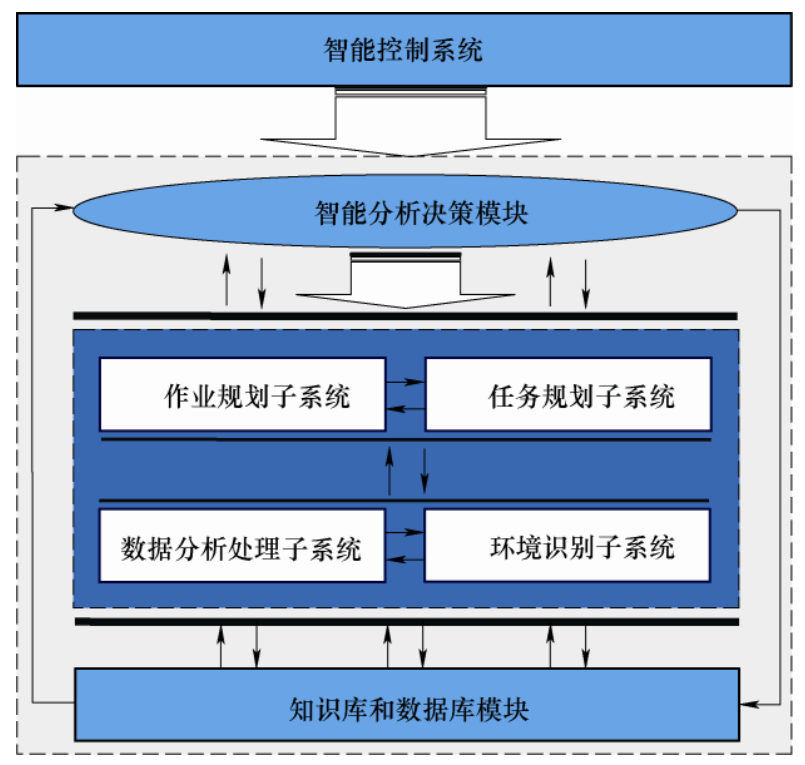

图 3 智能控制系统总体框架和主要数据流

\subsubsection{1 知识库和数据库模块}

知识库和数据库是智能分析决策模块的基础支 撑模块。知识库含有预先植入的经验数据和智能分 析决策模块产生的新知识等数据; 数据库数据包括 系统内置数据, 控制系统感知、分析、规划、决策 和执行数据以及系统自评、自学、自适应等过程产 生的需要存储在数据库中的数据。系统内置数据为 智能起重机产品开发、制造过程中保存在系统内部 和产品出厂时植入系统内部的数据。

人类在长期的学习和实践中积累了大量经验知 识, 这些经验知识构成了人的知识库, 每个人的知 识库结构和内容受人学习和实践的深度和广度等不 同而有所区别。当人遇到新问题时，首先会从既有 知识库中寻找解决新问题的方法, 如果知识库知识 可以解决新问题, 则自动调用知识库知识去解决新 问题; 如果知识库知识对解决新问题有启发但不能 完全解决问题, 人们会从知识库调取部分知识, 并 在此基础上完善解决新问题的方法; 如果知识库没 有任何知识可以用来解决新问题, 人就需要学习和 寻找全新的问题的解决方法, 新问题得到解决后会 将解决该新问题的方法植入到知识库。随着人的学 习和实践活动的不断深入, 知识库的积累也越丰富, 
解决新问题的能力也就越强。

智能起重机是具有理性行动和像人一样自主作 业能力的智能化设备, 面对新问题时会与人类一样 首先从系统的知识库搜寻解决方案, 知识库和数据 库的数据结构和数据量能帮助起重机解决新问题的 比例越多, 起重机用于寻找和解决新问题的方法所 占用的时间就越短, 智能起重机的作业效率和作业 敏捷程度就会越高, 智能起重机表现出的综合性能 就越好。知识库和数据库在起重机智能化作业中的 重要性可见一斑。

\subsubsection{2 智能分析决策模块}

智能分析决策模块是智能控制系统的核心，是 具有自学习、自决策和自适应能力的人工智能系统, 也是中央控制中心最终决策指令的具体规划者。该 模块利用感知并获取的大数据进行计算分析、深度 数据挖掘和推理演化及预测, 最终形成优化决策方 案和作业控制指令, 该指令通过通用控制系统实现 对智能起重机搬运作业过程的控制。智能分析决策 模块由数据分析处理、环境识别、任务规划和作业 规划 4 个子系统组成。

(1) 数据分析处理子系统。数据分析处理子系 统负责分析和处理上传到系统的所有数据, 负责根 据中央控制中心大数据传输和数据流向的要求, 按 规定格式准确传输分析和处理后的数据和信息, 需 要显示和管理的数据和图像信息将直接传送到广域 智能系统总控中心的人际交互管理层。经过数据分 析处理子系统分析和处理后的数据可以满足系统内 嵌的各智能规划和分析平台对数据质量和数据结构 的要求。

(2) 环境识别子系统。环境识别子系统使智能 起重机具有与人类相同功能的视觉, 通过处理感知 层获取的环境信息和感知数据, 可以准确提取智能 起重机作业环境中静止和动态移动物体的形状、大 小、位置、距离以及这些物体之间的相互关系和运 动状态等信息, 使智能起重机拥有三维动态环境地 图。环境识别子系统对环境动态变化的趋势具有智 能判断和分析对策能力。

(3) 任务规划子系统。任务规划子系统是智能 分析决策模块的核心。任务规划子系统内嵌货物搬 运顺序优化、搬运作业路径规划等多种专用的智能 规划软件和分析决策平台, 这些专用规划软件和平 台从数据分析处理子系统和环境识别子系统等获取 大量数据和信息, 通过采用大数据分析、智能算法 等人工智能技术, 对这些数据和信息进行计算、分 析和规划, 并完成与新任务完全匹配的优化作业方
案。这些各自相对独立的作业方案经过任务规划子 系统的统一规划和决策后, 形成针对新任务的完整 作业方案。

任务规划子系统可以预测起重机在执行上述作 业方案时可能会遇到的安全、效率等各类问题, 同 时根据环境条件的变化对上述作业方案和决策进行 不断完善、自主适应和实时更新。任务规划子系统 使智能起重机真正拥有了自预测、自学习、自评估、 自模拟、自决策、自适应、自补偿等人类才拥有的 智慧能力。

(4) 作业规划子系统。智能起重机在接到广域 智能系统总控中心或智能起重机人机交互管理层发 布的作业任务指令后, 智能控制系统首先从知识库 搜寻是否有完成指定任务的作业方案, 如果知识库 有满足该作业任务的成熟方案, 作业规划系统将直 接调用知识库方案, 结合系统内嵌的作业规划模型, 自动生成可执行的作业指令, 该指令被下传到通用 控制系统, 通过执行该作业指令完成该指定的作业 任务; 如果知识库没有或者尚无成熟的作业方案, 任务规划子系统立即开始工作, 借助专用的规划软 件和分析平台, 利用系统获取的大量有效数据和信 息，采用人工智能等技术形成针对新任务的完整方 案, 该作业方案被导入作业规划系统完成最终作业 指令的规划和输出。

作业规划子系统在正式输出作业决策指令前, 会结合任务规划子系统获取的作业方案预测结果, 对起重机作业过程进行虚拟模拟和作业决策指令的 预执行, 通过模拟过程的比较和评价, 对决策指令 进行必要修正, 最终输出完成新工作任务的起重机 搬运作业控制指令, 确保起重机与广域智能系统之 间的协同和精确配合作业, 使起重机在自适应状态 下能像人一样灵活机动地作业。

\section{2 感知层}

感知层的主要任务是实时感知起重机状态并 采集控制系统和人机交互管理等系统所需的各类 数据。

感知层由内感知和外感知两部分组成, 基本 构成与感知和控制数据流向如图 4 所示。外感知 从广域智能系统以及智能起重机以外部分感知并 获取数据, 内感知从智能起重机内部获取相关数 据。感知层通过各类传感器和专用感知系统实现 起重机状态感知与数据采集, 感知与传感系统精 度和技术水平将直接影响智能起重机的系统稳定 性和作业性能, 因此各类高端传感设备和技术的 研究开发值得重点关注。 


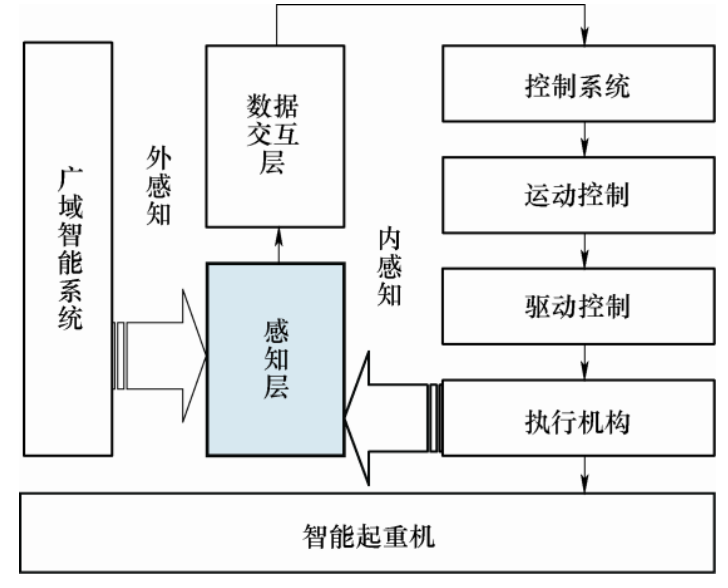

图 4 感知层基本构成与感知和控制数据流向

\section{3 数据交互层}

数据交互层是智能起重机控制系统各类数据的 接口层, 负责感知层采集到的内、外部数据等的上 传和控制系统最终作业指令数据等的下传。

感知层获取的感知数据有部分为无效数据, 为 了减少控制系统数据处理的压力, 提升控制系统运 行效率, 数据交互层通常会设置分控制器, 分控制 器可以将感知层采集到的数据进行预处理, 过滤掉 无效数据和非关键的信息和数据, 并将数据的格式 根据控制系统的要求进行调整, 最后数据交互层将 这些经过处理和调整后的数据通过总线等技术上传 到控制系统。

\section{4 人机交互管理层}

智能装备的智能化水平还远不能与人类相比, 正像组织中的人需要通过培训和管理不断提升其能 力和工作业绩一样, 智能装备同样需要持续不断地 进行系统升级和作业管理。人机交互管理层是人与 智能起重机实时进行交流、指令发布和信息交互的 管理平台, 也是人对智能起重机作业安全、作业质 量、维保状况、作业效率、作业能耗和作业成本等 作业状态进行监控和远程管理的平台。

\section{5 广域互联层}

信息互联互通是智能化最基本的要求, 智能起 重机是构成广域智能系统的关键智能设备之一, 因 此, 智能起重机不可能脱离开广域智能系统而独立 存在。智能起重机除了要建立内部信息共享和数据 互联互通机制外, 更需要和广域智能系统以及带有 数字特征的搬运物之间建立信息实时共享和数据互 联互通。因此, 智能起重机与广域智能系统的广域 数字互联成为实现智能起重机系统智能化作业的关 键, 而且广域信息共享和数据互联互通的深度和广 度决定了系统智能化的水平。

设置广域互联层的目的是希望在数据交互层基
础上强化智能起重机和广域智能系统内关连设备 之间的自我连接、自我作业行为和自我作业状态 的感知能力, 从而实现智能起重机内部及智能起 重机与广域智能系统之间保持有效的数据连接。 实现广域数据的互联互通是智能起重机具有的与 广域智能系统之间实时进行信息共享和数据交互 的能力, 是智能起重机有别于传统起重机的重要 特征之一。

\section{4 结论}

分析并总结了智能起重机的关键特性, 提出了 智能起重机控制系统的总体架构, 并对核心功能模 块和子系统进行了分析介绍。

（1）定义起重机广域智能系统, 包括智能起重机 在内的广域智能系统的群体智能化是实现智能起重 机系统智能化作业的关键。

（2）智能起重机最重要的个性化特征就是, 使起 重机最大可能地满足广域智能系统对起重机提出的 个性化作业和智能化要求。

（3）智能起重机比较典型的通用性关键特征包 括监测感知、数据互联、学习分析、优化决策、实 时反馈、自适应、理性行动和像人一样自主作业等。

（4）智能起重机控制系统总体架构由中央控制 中心和感知层、数据交互层、人机交互管理层及广 域互联层组成。任务规划子系统是智能起重机中央 控制中心的智慧大脑，该子系统内嵌货物搬运顺序 优化、搬运作业路径规划等多种专用的智能规划软 件和分析决策平台, 为起重机作业提供智能化解决 方案并实时预测起重机在执行作业指令过程中可能 会遇到的安全、效率等问题, 实现起重机的自主控 制和自适应作业。

(5) 数据是认识并最终解决起重机智能化控制 中非模式、不确定性问题的基础, 可以发现凭经验 无法发现或意识不到的影响起重机装卸作业的因素 和各影响因素之间存在的相互关联, 可以降低认知 的不确定性。强大的数字互联能力是支撑并实现起 重机智能化的基础。

(6) 智能起重机控制系统的知识库和数据库的 数据结构和数据量能帮助起重机解决新问题的比例 越多，起重机用于寻找和解决新问题的方法所占用 的时间就越短，智能起重机的作业效率和作业敏捷 程度就会越高, 智能起重机表现出的综合性能就 越好。

(7) 起重机智能化研究应首先聚焦自动化发展 
水平比较高的行业, 以及这些行业智能化建设对起 重机产品类别和智能化水平的具体需求, 可以强化 起重机智能化研究的针对性和目的性。

随着我国从制造大国向制造强国的快速推进, 传统制造业正在快速转型并逐步迈向智能制造新时 代, 制造业大环境的发展将同步带动起重机行业从 传统产业向智能化方向的发展。希望与行业科技工 作者一起推动起重机智能化转型升级和起重机产品 的智能化发展, 相信在全行业科研工作者不解努力 和艰苦攻关下, 我国起重机行业能早日迎来智能化 时代。

\section{参 考 文 献}

[1] 制造强国战略研究项目组. 制造强国战略研究. 智能制 造专题卷 $[\mathrm{M}]$. 北京: 中国工信出版集团, 电子工业出 版社, 2015 .

Project Group on Strategic Research of Manufacturing Powerful Countries. Special volume on intelligent manufacturing of strategic research of manufacturing powerful countries[M]. Beijing : China Industry and Telecommunications Publishing Group , Electronic Industry Publishing House, 2015.

[2] 宓为建, 张志伟, 宓超. 基于机器视觉的集装箱锁孔识 别算法研究 [J]. 中国工程机械学报, 2016, 14(5): 399-402.

MI Weijian, ZHANG Zhiwei, MI Chao. Study oncontainer hanging holes recognition algorithm based on machine vision[J]. Chinese Journal of Construction Machinery, 2016, 14(5): 399-402.

[3] 杨宇华, 张氢, 聂飞龙. 集装箱自动化码头发展趋势分 析[J]. 中国工程机械学报, 2015, 13(6): 571-576.

YANG Yuhua, ZHANG Qing, NIE Feilong. Trend analysis on automation container terminals[J]. Chinese Journal of Construction Machinery, 2015, 13(6): 571-576.

[4] 赵斌, 闻佳华, 周颖达. 虚拟世界中的自动化桥式起重 机[J]. 起重运输机械, 2019(15): 83-86.

ZHAO Bin, WEN Jiahua, ZHOU Yingda. Automatic bridge crane in virtual world[J]. Hoisting and Conveying Machinery, 2019(15): 83-86.

[5] 姜广路. 集装箱港口内陆堆场起重机的自动化[J]. 起 重运输机械, 2018(6): 153-155.

JIANG Guanglu. The automation of crane in inland storage yard of container port[J]. Hoisting and Conveying Machinery, 2018(6): 153-155.

[6] 梁承姬, 林洋. 自动化码头双小车岸桥与 $\mathrm{AGV}$ 协调调
度问题研究 $[J]$. 计算机工程与应用, 2019, 55(10): 256-263.

LIANG Chengji, LIN Yang. Research on problem of double-trolley quay crane and AGV coordinated scheduling in automated terminal[J]. Computer Engineering and Applications, 2019, 55(10): 256-263.

[7] 王晓帅, 张氢, 张小龙. 自动化集装箱码头轨道式龙门 起重机远程作业系统的实现 [J]. 自动化仪表, 2016, 37(2): 33-40.

WANG Xiaoshuai, ZHANG Qing, ZHANG Xiaolong. Implementation of the remote operation system for rail mounted gantry crane in automated container terminal[J]. Process Automation Instrumentation, 2016, 37(2): 33-40.

[8] 李国杰. 起重机摆动控制系统研究[J]. 起重运输机械, 2012(9): 1-6.

LI Guojie. Research on crane swing control system[J]. Hoisting and Conveying Machinery, 2012(9): 1-6.

[9] 李国杰. 考虑起吊物初始摆动的起重机摆动控制技 术 $[J]$. 起重运输机械, 2012(10): 5-12.

LI Guojie. Sway control technology for crane by considering primitive sway of object[J]. Hoisting and Conveying Machinery, 2012(10): 5-12.

[10] 李永, 李钢强. 某型全自动起重机在核电厂电机组装中 的应用[J]. 中国设备工程，2019(9)：139-141.

LI Yong, LI Gangqiang. Application of fully automatic crane in the assembly of nuclear power plant motor[J]. China Plant Engineering, 2019(9): 139-141.

[11] 北森. クレーンの最適転法 $[C] / /$ 第 3 回計測自動制御学 会学術講演会予稿集, 1964: 347-348.

KITA M. Optimum rolling of crane[C]//Proceedings of the 3rd Conference of Japan Institute of Automatic Measurement and Control, 1964: 347-348.

[12] 森下螈. 走行クレーン運転自動化のための振れ止め 制御 [J]. 計測自動制御学会論文集, 1978, 14(6): 739-744.

MORISHITA I. A new control algorithm for the grab-swing elimination in the automatic operationg cranes[J]. Transactions of the Japan Institute of Automatic Measurement and Control, 1978, 14(6): 739-744.

[13] 近藤良, 武田亭, 田口彦衛. 振動周期に基づく旋回ク レーンの振れ止め制御[J]. 日本机械学会论文集( C 編), 2001, 67(655): 725-731.

KONDO R, TAKEDA T, TAGUCHI H. Sway control of rotary crane based on load swing period[J]. Transactions of the Japan Society of Mechanical Engineers (Part C), 
2001, 67(655): 725-731.

[14] 小島宏行, 羽廣賢一. 遺伝的アルゴリズムを用いた直 進形クレーンの最適軌道計画と残留振れ仰制制御の 実験[J]. 日本机械学会论文集 (C 編)，2003，69(682): 1479-1485.

KOJIMA H, HABIRO K. Optimal trajectory planning of a travelling crane using with genetic algorithm and residual vibration reduction control experiments[J]. Transactions of the Japan Society of Mechanical Engineers (Part C), 2003， 69(682): 1479-1485.

[15] SMOCZEK J, SZPYTKO J, HYLA P. The application of an intelligent crane control system[C]//Proceedings of 13th IFAC Symposium on Control in Transportation Systems. Sofia: The International Federation of Automatic Control, 2012: 280-285.

[16] ROGER M, LUIS T A. A family of anti-swing motion controllers for $2 \mathrm{D}$-cranes with load hoisting/lowering[J]. Mechanical Systems and Signal Processing, 2019, 133: 106253-106271.

[17] MAGHSOUDI M J, RAMLI L, SUDIN S, et al. Improved unity magnitude input shaping scheme for sway control of an underactuated 3D overhead crane with hoisting[J]. Mechanical Systems and Signal Processing, 2019, 123 : 466-482.

[18] ANDRES B, VINICIUS P, HAMID R, et al. Novel control method for overhead crane's load stability[J]. Procedia Engineering, 2015, 106: 108-125.

[19] RAMLI L, MOHANED Z, AUWALU M, et al. Control strategies for crane systems: A comprehensive review[J]. Mechanical Systems and Signal Processing, 2017, 95: $1-23$.

[20] KANESHIGE A, NAGAI S, UEKI S, et al. Development of the autonomous overhead travelling crane with real time path-planning based on obstacle information[C]// Proceedings of 13th IFAC Symposium on Control in Transportation Systems. Sofia : The International Federation of Automatic Control, 2012: 30-36.

[21] RINCON L, KUBOTA Y, VENTURE G, et al. Inverse dynamic control via "simulation of feedback control" by artificial neural networks for a crane system[J]. Control Engineering Practice, 2020, 94: 1-18.

[22] DUONG S C, UEZATO E, KINJO H, et al. A hybrid evolutionary algorithm for recurrent neural network control of a three-dimensional tower crane $[\mathrm{J}]$. Automation in Construction, 2012, 23: 55-63.
[23] SZPYTKO J, DUARTE Y S. Digital twins model for cranes operating in container terminal[J]. International Federation of Automatic Control，2019， 52(10): 25-30.

[24] 汪首坤, 郭俊杰, 王军政, 等. 基于混合群智能优化的 机器人立体视觉标定 $[J]$. 机械工程学报, 2012, 48(1): 57-63.

WANG Shoukun, GUO Junjie, WANG Junzheng, et al. Robot stereo vision calibration based on hybrid swarm intelligent optimization[J]. Journal of Mechanical Engineering, 2012, 48(1): 57-63.

[25] 季旭全, 王君臣, 赵江地, 等. 基于机器人与视觉引导 的星载设备智能装配方法 $[\mathrm{J}]$. 机械工程学报, 2018, 54(23): $63-72$.

JI Xuquan, WANG Junchen, ZHAO Jiangdi, et al. Intemgent robotic assembly method of spaceborne equipment based on visual guidance[J]. Journal of Mechanical Engineering, 2018， 54(23): 63-72.

[26] 刘久富, 陈鬼, 苏青琴, 等. 基于 Markov 对策的码垛 机器人三维路径规划 [J]. 中国机械工程, 2012, 23(7): 851-855.

LIU Jiufu, CHEN Kui, SU Qingqin, et al. Markov game based 3D path planning for palletizing $\operatorname{robot}[\mathrm{J}]$. China Mechanical Engineering, 2012， 23(7): 851-855.

[27] 史也, 梁斌, 王学谦, 等. 基于量子粒子群优化算法的 空间机器人非完整笛卡尔路径规划 $[\mathrm{J}]$. 机械工程学报, 2011, 47(23): 65-73.

SHI Ye, LIANG Bin, WANG Xueqian, et al. Cartesian non-holonomic path planning of space robot based on quantum-behaved particle swarm optimization algorithm [J]. Journal of Mechanical Engineering, 2011，47(23): 65-73.

[28] LEGASHEV L V, LETUTA T V, POLEZHAEV P N, et al. Monitoring certification and verification of autonomous robots and intelligent systems: Technical and legal approaches[J]. Procedia Computer Science, 2019, 150: $544-551$.

[29] 贾庆轩, 陈钢, 孙汉旭, 等. 基于 $A^{*}$ 算法的空间机械臂 避障路径规划 [J]. 机械工程学报, 2010, 46(13)： 109-115.

JIA Qingxuan, CHEN Gang, SUN Hanxu, et al. Path planning for space manipulator to avoid obstacle based on $\mathrm{A}^{*}$ algorithm[J]. Journal of Mechanical Engineering, 2010, 46(13): 109-115.

[30] 李运华, 何刘宇, 杨丽曼. 非完整约束多轴驱动车辆的 路径跟踪控制[J]. 机械工程学报，2014，50(5)：33-41. 
LI Yunhua, HE Liuyu, YANG Liman. Path-following control of nonholonomic constraint multi-axle drive vbhicle[J]. Journal of Mechanical Engineering, 2014, 50(5): $33-41$.

[31] 张利鹏, 王胜, 袁心茂. 分布式驱动智能汽车对开坡道 起步双重转向控制[J]. 机械工程学报, 2019, 55(20): 205-213.

ZHANG Lipeng, WANG Sheng, YUAN Xinmao. Dual steering control of distributed drive intelligent vehicle start on split-slope $\operatorname{road}[\mathrm{J}]$. Journal of Mechanical Engineering, 2019, 55(20): 205-213.

[32] 江浩斌, 沈峥楠, 马世典, 等. 基于信息融合的自动泊 车系统车位智能识别 [J]. 机械工程学报, 2017 , 53(22): 125-133.

JIANG Haobin, SHEN Zhengnan, MA Shidian, et al. Intelligent identification of automatic parking system based on information fusion[J]. Journal of Mechanical Engineering, 2017, 53(22): 125-133.

[33] 章仁綌, 熊璐, 余卓平. 智能汽车转向轮转角主动控 制[J]. 机械工程学报，2017，53(14): 106-113.

ZHANG Renxie, XIONG Lu, YU Zhuoping. Active steering angle control for intelligent vehicle[J]. Journal of Mechanical Engineering, 2017, 53(14): 106-113.

[34] 王跃飞, 黄斌, 吴源, 等. 面向智能汽车的 Ethernet AVB 网络设计与优化 $[\mathrm{J}]$. 机械工程学报, 2019, 55(6): 166-177.

WANG Yuefei, HUANG Bin, WU Yuan, et al. Design and optimization of Ethernet AVB network for intelligent vehicle[J]. Journal of Mechanical Engineering, 2019, 55(6): 166-177.

[35] 牛礼民, 杨洪源, 周亚洲, 等. 混合动力汽车动力总成 多智能体集成控制策略[J]. 机械工程学报, 2019, 55(12): 168-188.

NIU Limin, YANG Hongyuan, ZHOU Yazhou, et al. Hybrid electric vehicle integrated control strategy based on multi-agent[J]. Journal of Mechanical Engineering, 2019, 55(12): 168-188.

[36] 王艺, 蔡英风, 陈龙, 等. 基于模型预测控制的智能网 联汽车路径跟踪控制器设计 $[J]$. 机械工程学报, 2019, 55(8): 136-144.

WANG Yi, CAI Yingfen, CHEN Long, et al. Design of intelligent and connected vehicle path tracking controller based on model predictive control[J]. Journal of Mechanical Engineering, 2019, 55(8): 136-144.

[37] BAHNES N, KECHAR B, HAFFAF H. Cooperation between intelligent autonomous vehicles to enhance container terminal operations[J]. Journal of Inovation in Digital Ecosystems, 2016(3): 22-29.

[38] 熊振华, 孙宇昕, 丁龙杨. 智能车床的颤振实时辨识与 在线抑制系统研究 [J]. 机械工程学报, 2018, 54(17): 85-93.

XIONG Zhenhua, SUN Yuxin, DING Longyang. Online chatter detection and suppression system for intelligent machine tool[J]. Journal of Mechanical Engineering, 2018, 54(17): 85-93.

[39] 黄莹, 卢秉恒, 赵万华. 云计算在智能机床控制体系中 的应用探析[J]. 机械工程学报, 2018, 54(8): 210-216. HUANG Ying, LU Bingheng, ZHAO Wanhua. Application of cloud computing in intelligent machine tool control system[J]. Journal of Mechanical Engineering, 2018, 54(8): 210-216.

[40] 刘献礼, 刘强, 岳彩旭, 等. 切削过程中的智能技术 [J]. 机械工程学报，2018，54(16): 46-61.

LIU Xianli, LIU Qiang, YUE Caixu, et al. Intelligent machining technology in cutting process $[\mathrm{J}]$. Journal of Mechanical Engineering, 2018，54(16): 46-61.

[41] 陈雪峰, 张兴武, 曹宏瑞. 智能主轴状态监测诊断与振 动控制研究进展[J]. 机械工程学报, 2018，54(19): 58-69.

CHEN Xuefeng, ZHANG Xingwu, CAO Hongrui. Advances in condition monitoring diagnosis and vibration control of smart spindles[J]. Journal of Mechanical Engineering, 2018, 54(19): 58-69.

[42] 崔云先, 张博文, 丁万昱, 等. 瞬态切削用智能测温刀 具的研究 [J]. 机械工程学报, 2017, 53(21): 174-180. CUI Yunxian, ZHANG Bowen, DING Wanyu, et al. Research on the cutting tool with intelligent transient temperature measuring system[J]. Journal of Mechanical Engineering, 2017, 53(21): 174-180.

[43] 姜洪开, 邵海东, 李兴球. 基于深度学习的飞行器智能 故障诊断方法[J]. 机械工程学报, 2019，55(7): 27-34. JIANG Hongkai, SHAO Haidong, LI Xingqiu. Deep learning theory with application in intelligent fault diagnosis of aircraft[J]. Journal of Mechanical Engineering, 2019, 55(7): 27-34.

[44] 康荣杰, 杨铖浩, 杨名远, 等. 会思考的机器一机械智 能[J]. 机械工程学报, 2018，54(13): 15-24.

KANG Rongjie, YANG Chenghao, YANG Mingyuan, et al. Machines which can think: Mechanical intelligence [J]. Journal of Mechanical Engineering, 2018, 54(13): 
$15-24$.

[45] 刘强. 智能制造理论体系架构研究[J]. 中国机械工程, 2020, 31(1): 24-36.

LIU Qiang. Study on architecture of intelligent manufacturing theory[J]. China Mechanical Engineering, 2020, 31(1): 24-36.

[46] LEE J, BAGHERI B, JIN Chao. Introduction to cyber manufacturing[J]. Manufacturing Letters, 2016(8): 11-15.

[47] WANG B. The future of manufacturing: A new
perspective[J]. Engineering, 2018, 4(5): 722-728.

[48] 上海港机重工有限公司. 港口起重机设计规范 [M]. 北 京：人民交通出版社， 2007 .

Shanghai Port Machinery Heavy Industry Co., Ltd. Design rules for port cranes[M]. Beijing : China Communications Press, 2007.

作者简介: 李国杰, 男, 1963 年出生, 教授。主要研究方向为起重运输 机械及智能化。

E-mail: ligjsh021@126.com 